\title{
"Los ceros también valen". Conocimientos de alumnos de sexto grado de primaria sobre el cero como elemento del sistema decimal
}

\author{
"Zeros are also worth." Knowledge about zero in System \\ of Decimal Numbering in sixth grade students
}

\author{
Olivia Ávalos Esparza ${ }^{1}$ \\ Diana Violeta Solares Pineda²
}

\begin{abstract}
Resumen: La apropiación del cero en los años escolares trascurre por un camino complejo. Los alumnos tienen que confrontar conocimientos contradictorios, como representar la ausencia de cantidad mediante "una presencia" (el signo cero). Estos obstáculos fueron enfrentados por la humanidad para construir el concepto del cero. En el presente artículo reportamos las funciones que un grupo de 10 estudiantes de sexto grado de primaria le atribuyen al cero dentro del sistema de numeración decimal. Tales conocimientos se pusieron de manifiesto al resolver sumas horizontales, diseñadas con base en ciertas variables didácticas. Las respuestas de los alumnos dan cuenta de las siguientes funciones asignadas al cero: a) Es indicador de cómo sumar; b) Ayuda como diferenciador de cantidades; c) Es un signo que representa ausencia. La mayor concentración de respuestas está dentro de esta última categoría (45.17\%). Los resultados obtenidos aportan a las investigaciones didácticas y psicológicas relativas a cómo los niños de distintas etapas escolares se apropian del sistema
\end{abstract}

Fecha de recepción: 26 de octubre de 2017. Fecha de aceptación: 04 de noviembre de 2018.

1 Universidad Autónoma de Querétaro, Facultad de Psicología, oliviava25@hotmail.com, orcid.org/00000002-2558-3391.

2 Universidad Autónoma de Querétaro Facultad de Psicología, violetasolares@gmail.com, orcid.org/00000001-6034-6693. 
de numeración decimal, particularmente en sexto grado, donde se espera que diversos conocimientos sobre el sistema ya estén consolidados.

Palabras clave: conocimiento escolar; variables didácticas; funciones del cero; sexto grado de primaria; cálculos aritméticos.

\begin{abstract}
The zero appropriation during school years passes through a complicated path. Students have to deal with contradictory knowledge such as representing the absence of quantity by "a presence" (the zero sing). Humanity faced these obstacles to build the concept of zero. In this paper we report usages and functions that a group of 10 students of elementary school attributed to the zero within Decimal Number System. That knowledge emerged while solving an arithmetic calculation that was previously designed based on certain didactic variables. Answers displayed three kind of knowledge related to the zero: a) As how-to-add indicator; b) As quantity distinction and c) As a sign to represent absence. The highest frequency of answers was found into the last class (45.17\%). Obtained results contribute to the didactic and psychological research related to scholar children appropriation of Decimal Number System, especially in sixth grade, where it is expected that knowledge about it is established already.
\end{abstract}

Keywords: school knowledge; didactic variables; zero; sixth grade elementary school; arithmetic calculations.

\title{
INTRODUCCIÓN
}

El sentido del cero dentro de las matemáticas ha evolucionado a lo largo de la historia. En sistemas de numeración posicionales tan antiguos como el babilonio, alrededor del siglo III a. C. se usaba una especie de cero primitivo (un pequeño círculo colocado entre dos cifras) que evitaba confusiones en la interpretación de cantidades escritas. Por ejemplo, si quisiéramos escribir el número 103, podríamos hacerlo usando el círculo en lugar del cero, de esta manera: 103 (D'Amore y Fandiño, 2014).

Existían variantes gráficas de dicho cero, entre ellas ganchos y cuñas inclinadas, las cuales se interpretaban como "no hay nada en esta columna". Su función era equivalente a la de signos de puntuación. Además, este tipo de 
ceros sólo eran usados en medio de las escrituras numéricas y nunca al final pues, después de todo, su función era únicamente separar números para leerlos correctamente. Kaplan (2004) indica que existían diversas representaciones gráficas para el cero, debido a que su forma y función no estaban establecidas de manera definitiva. Transcurrirían algunos milenios para que el cero formara parte de la estructura de los sistemas posicionales y fuera concebido como número nulo.

Así, en el 500 d. C, el astrónomo hindú Aryabatha, buscando una forma de registrar números grandes, estructuró un sistema de escritura numérico donde figura el kha, que se traduce como "lugar". Esta palabra dio un primer nombre al cero, cuya intención era señalar simultáneamente una ausencia y marcar una posición que podría ser ocupada por otros números. Esta distinción es relevante porque desde ese momento el cero ya no fue usado solamente como marca de puntuación que auxilia para interpretar una cantidad, sino que también era "un vacío receptor" que con su presencia otorgaba valor a otros números (Kaplan, 2004).

Cabe señalar que el cero seguirá siendo auxiliar para diferenciar cantidades, pero a partir de las aportaciones de Aryabatha y otros sabios hindúes empezó a cumplir otra función: representar ausencia de un agrupamiento decimal. Ambos aspectos son importantes, pero cualitativamente diferentes. Por ejemplo, el cero de 103 ayuda a distinguir entre las cantidades 103 y el 13, y también representa la ausencia de la agrupación de decenas. Cuando el cero se escribe para indicar ese vacío receptor, se llama cero como marca de posición. Estas dos funciones han sido respuestas de la humanidad para enfrentar el reto de comunicar ideas numéricas de manera clara y sucinta.

Como es notable, esas funciones aparecen alejadas una de la otra durante un extenso periodo y su sentido como objeto matemático seguiría evolucionado. Hacia el año 628 d. C., el matemático y astrónomo Brahmagupta dejó registro de cómo efectuar las operaciones de suma, resta, potencias, raíces, etc. Este hecho implicó dotar del sentido de "número nulo" al cero. De esta manera, los sabios hindúes reunieron dos nociones complejas del cero: la de marca de posición y la de nulidad. Con ello, el cero como número permitió el desarrollo del álgebra y desempeñó un papel fundamental en otras ramas de las matemáticas (Ifrah, 1987).

Encontramos en la actualidad propuestas que describen los diferentes roles del cero. De acuerdo con Blake y Verhille (1985), el cero ha tenido diversas funciones, entre las cuales recuperamos las siguientes: 
a) Cero como placeholder: permite guardar un espacio vacío y fue particularmente útil para el registro e interpretación de cantidades y la distinción entre números como 52, 520, 502. Fue usado por culturas como la babilónica y la maya.

b) Cero como variable: se refiere al punto "sunya" de los hindúes, usado para representar lo desconocido en una ecuación, hasta que sea ocupado por un número determinado. Coincide con la función de "vacío receptor" señalada por Kaplan (2004).

c) El cero como space filler: los autores identifican distintos usos en esta categoría. En términos generales, es el uso del cero para redondear cantidades o para ocupar las columnas que quedan sin cifras al momento de resolver algoritmos convencionales (Figura 1, tomada de Blake y Verhille, 1985: 39).

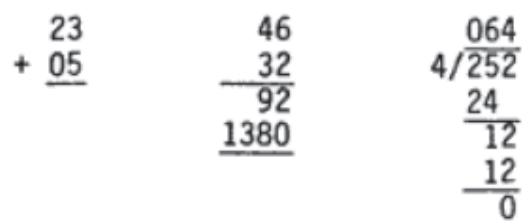

Figura 1. El cero como space filler. Los ceros se escriben en las columnas de los algoritmos para llenar los espacios vacíos, donde no es necesario el cero para interpretar el número.

Estos autores señalan que algunas de esas funciones se mantienen en prácticas escolares. También afirman que algunas de ellas son indistinguibles si se les considera de una manera superficial, por lo que es necesario analizar el contexto donde el cero escrito es usado, para así tener una comprensión amplia de su estructura matemática.

Por su parte, D’Amore y Fandiño (2014) plantean que, según el área matemática donde se le ubique, el cero puede tener la función de cifra, número cardinal y de número ordinal. Sobre el cero cifra, los autores indican: "si consideramos un sistema posicional cualquiera, se llama cero a una de las cifras, aquella que sirve para indicar un espacio vacío en la escritura polinominal" (D’Amore y Fandiño, 2014: 15). Respecto al cero cardinal, señalan que es el cardinal de un conjunto vacío. Estas definiciones pueden ser compatibles con las de cero como vacío receptor y el cero que representa ausencia que aparecen en nuestra revisión histórica. 
La diversidad de funciones del cero y las aparentes contradicciones entre algunas de ellas son retos que la humanidad tuvo que enfrentar en el transcurso de elaboración de este concepto. Incluso, el lenguaje que usamos actualmente en el contexto escolar (tanto en nivel básico como superior) es un reflejo de las dificultades históricas que ha tenido la humanidad en dicha elaboración (Blake y Verhille, 1985). Tales dificultades guardan cierta proximidad con las que experimentan los niños en sus procesos de apropiación del cero como número y, particularmente, como elemento sustancial del sistema de numeración decimal.

Hay diversas investigaciones que abordan la construcción del concepto del cero por parte de niños en la etapa preescolar. Como mostraremos más adelante, las aportaciones de esos trabajos han sido sumamente relevantes para investigaciones posteriores y para el diseño didáctico. Sin embargo, poco sabemos sobre cómo esas concepciones evolucionan a lo largo de la escolaridad básica, pues la mayoría de las exploraciones están centradas en niños pequeños. Se hace necesario, entonces, analizar el papel que la escuela juega en esos aprendizajes: ¿En qué medida ha contribuido -o no- en la transformación paulatina de los conocimientos matemáticos de los alumnos? (en este caso, en la transformación de las nociones iniciales del cero).

De acuerdo con Martí (2005) existen conocimientos del sistema decimal que, por su complejidad, sólo se logran adquirir completamente mediante la enseñanza formal. En el currículo oficial de la educación primaria de México identificamos que, si bien el cero como objeto matemático no es estudiado de manera sistemática, a través de las orientaciones didácticas ofrecidas a los docentes (SEP, 2011), se proponen actividades de reflexión en torno al cero cuando el niño está aprendiendo a escribir números con cero intermedio. Asimismo, en los grados superiores de primaria se sugiere comparar similitudes y diferencias entre sistemas posicionales y no posicionales, al igual que entre el sistema de numeración oral y el escrito.

En este artículo presentamos los conocimientos sobre el cero que manifestaron 10 alumnos de sexto grado de primaria al resolver determinados cálculos aritméticos. Nuestro punto de partida es que, aun cuando el currículo de la educación primaria en México no plantea una ruta específica para el tratamiento de este número, los estudiantes tienen conocimientos que, al parecer, han construido a partir de su interacción con los números naturales, con el sistema de numeración decimal y con los cálculos aritméticos. Tales conocimientos podrían estar influenciados tanto por las hipótesis que los niños han hecho respecto al 
cero, así como por las actividades y los contenidos con los que han interactuado en su trayecto escolar.

Cabe aclarar que lo que aquí presentamos es parte de un estudio más amplio (Ávalos, 2016) en el que nos propusimos identificar los conocimientos de esos alumnos respecto al valor posicional. Para ello les planteamos ciertas tareas de cálculo aditivo; una de las consignas que les dimos fue que describieran la función que le atribuyen al cero al resolver dichas tareas. Centrándonos exclusivamente en ese bloque de resultados de la investigación original, desarrollamos lo que aquí exponemos.

Los objetivos de este trabajo son, en primer lugar, reportar las funciones atribuidas al cero por niños de sexto grado cuando resolvían cálculos y, en segundo lugar, analizar el papel de ciertas variables didácticas en los procedimientos de solución de los alumnos.

Las variables didácticas que caracterizan nuestro instrumento, de las cuales queremos conocer el impacto sobre los procedimientos de los niños, son: orden de los sumandos, ausencia de algún orden, agrupamiento decimal y cero en el resultado.

Presentamos, en primer lugar, algunas investigaciones sobre cómo los estudiantes interpretan al cero. La revisión de estos antecedentes nos permitió construir un marco de interpretación para los resultados de nuestra propia investigación.

Describimos, posteriormente, la metodología de investigación: el diseño, la muestra y el instrumento. Después se exponen los resultados, su interpretación y la discusión que establecimos con los estudios de referencia. Por último, en las conclusiones desatacamos las respuestas a las preguntas de la investigación y los nuevos cuestionamientos que surgen a la luz de nuestros resultados.

\section{ESTUDIOS QUE INDAGAN CONCEPCIONES DEL CERO EN NIÑOS}

Diversas investigaciones reportan lo que los niños saben sobre el cero, ya sea como número o como elemento del sistema de numeración decimal. Presentamos un panorama de las mismas.

Investigaciones pioneras sobre concepciones del cero reportan que los niños en etapa preescolar poseen conocimientos que se traducen en frases como "los ceros no son números" o que "no son nada" (Leeb-Lumberg, 1977; Lovell, 1971). Estos autores denominan esos saberes misconceptions (concepciones erróneas) 
y afirman que la prevalencia de los problemas en el aprendizaje de las matemáticas se debe a ellas (Wheeler y Feghali, 1983; Wheelwe, 1987; Carpenter, Franke y Levi, 2002, citados en Anthony y Walshaw, 2004).

Otro conjunto de trabajos tiene una apreciación distinta de los conocimientos identificados en los alumnos de preescolar. Trabajos como los de Hughes (1986) indican que los niños de esa misma etapa conocen el cero, saben su nombre y pueden interpretar que representa ausencia de cantidad. Sin embargo, eso no implica que puedan utilizar la notación del cero para representar dicha ausencia, como lo documentan García-Milà, Teberosky y Martí (2000).

Por su parte, Wellman y Miller (1986) reportan que niños preescolares llegan a comprender que el cero es un número entre los demás, cuyo valor es "nada". Consideran que ello sienta las bases para la construcción de posteriores conocimientos algebraicos.

Los trabajos de investigación citados tienen en común haber indagado conocimientos sobre el cero como número. De acuerdo con los últimos, los niños desde pequeños pueden interpretar que el cero representa ausencia. Parecen coincidir con que estos conocimientos tempranos sientan las bases para el desarrollo de saberes matemáticos posteriores. Cabe preguntarnos si esa interpretación despliega nuevos significados y avanza hasta la noción de representar ausencia de una posición determinada cuando se trata de analizar el cero en la estructura del sistema de numeración decimal.

En ese sentido, un conjunto de estudios más recientes reporta los conocimientos del cero que emergen en exploraciones cuyo objetivo es, más ampliamente, el sistema de numeración decimal (Lerner, 1992, 1994, 2005; Scheuer, Sinclar, Merlo de Rivas, Tièche, 2000; Wolman y Ponce, 2013).

En lo que respecta a la escritura e interpretación de números, encontramos investigaciones que documentan las dificultades que tienen niños mayores para escribir números "grandes". Un trabajo llevado a cabo por Brown (1981) con alumnos de nivel secundaria informa que sólo la mitad de los entrevistados pudieron escribir correctamente el número "400,073" (four hundred thousand and seventy-three, dado que es un estudio de habla inglesa). Resultados similares se reportaron en el trabajo de Crooks y Flockton (2002), quienes señalan que de 2,869 estudiantes, solamente 50\% de los mayores de 8 años pudieron escribir correctamente el número "200,043".

Sin embargo, estos trabajos no indagan sobre las justificaciones que los estudiantes ofrecen a sus escrituras numéricas. Por el contrario, trabajos como los de Lerner (1994), Scheuer, Sinclar, Merlo de Rivas, Tièche, (2000), Wolman y 
Ponce (2013) y Centurión y Saiz (2014) sí indagan acerca de las razones que justifican la manera no convencional de escribir números. Estos investigadores sostienen que los niños han construido hipótesis sobre el funcionamiento del sistema de numeración decimal, un hecho que se traduce en reglas de acción que les permiten interpretar y producir números cuya escritura convencional desconocen.

Entre ellas destacan la regla de acción construida por los niños que dicta que si se quiere tener un número grande, del orden de los millones, deben poner más ceros en una escritura numérica (Centurión y Saiz, 2014). También se reporta la relevancia que cobra la coma en números mayores (como 3,041,100) para ordenar, separar y diferenciar cantidades. Al respecto, una de las reglas de acción de los alumnos mayores es que cambiar de lugar la coma en una escritura numérica puede hacer que ésta cambie su valor. Es decir, los entrevistados elaboraron dos escrituras numéricas que sólo se distinguían entre sí por la ubicación de la coma, pero les asignan valores diferentes. Por ejemplo, escribían 10,000 para diez mil y 1,0000 para un millón. La hipótesis subyacente es que, al cambiar la coma de lugar, cambia el número ${ }^{3}$ (Wolman y Ponce, 2013). Si bien no todas esas reglas de acción permiten la escritura correcta de números, es fundamental reconocer que estos errores forman parte de todo proceso constructivo de conocimiento. Según la Teoría de las Situaciones Didácticas (en adelante TSD) los errores son una condición característica, coherente, aunque no correcta, de un conocimiento (Brousseau, 2007).

En esta misma línea de investigación resultó relevante para nuestro trabajo el estudio efectuado por Lerner (1992) sobre la función del cero en escrituras numéricas, con niños de primero, tercero y quinto grado de primaria.

La autora planteó una tarea de lectura de números en la cual los alumnos tenían que señalar si el cero vale y qué papel juega en escrituras numéricas. Llega a la conclusión de que los niños han construido reglas de acción para interpretar el cero de acuerdo con su grado de escolaridad. De manera general, enumeramos los hallazgos a continuación.

a) Los alumnos de primer grado de primaria indican que el cero no tiene valor, pero no puede suprimirse cuando aparece a la derecha de un

\footnotetext{
${ }^{3}$ Cabe aclarar que en la investigación referida, originalmente se habla de punto y no de coma, ya que en Argentina -y en otros países- el punto tiene la función que en México asignamos a la coma (para separar cifras en los números enteros).
} 
número. Por ejemplo, un 10 deja de ser diez si eliminamos el 0 a la derecha del 1.

b) Niños de tercero y quinto saben que el valor del cero depende de su ubicación en la cadena de números. Argumentan que, aunque el cero no vale por sí mismo, es necesario escribirlo. Para ellos el cero sirve como diferenciador de cantidades: los ceros valen en tanto que sirven para diferenciar números. Ejemplo: si no estuviera un 0 en 103, sería el número trece y no el ciento tres.

c) Según la autora, estos entrevistados han comprendido que el cero escrito en una cantidad indica que no hay elementos correspondientes a esa potencia de base. Sus hallazgos contribuyen a considerar que la reconstrucción del cero dentro del sistema posicional es lenta y compleja. En muchos casos, los alumnos pueden hacer uso del cero sin que lleguen necesariamente a explicitar sus funciones en términos convencionales.

Como puede apreciarse, hay diversas investigaciones que abordan la construcción del concepto del cero por parte de niños en la etapa escolar, y las aportaciones de esos trabajos han sido sumamente relevantes para investigaciones posteriores y para el diseño didáctico. Sin embargo, salvo por el trabajo de Lerner (1992), poco sabemos sobre cómo esas concepciones evolucionan a lo largo de la escolaridad básica, pues las exploraciones, en su mayoría, están centradas en niños pequeños.

\section{MARCO TEÓRICO}

De acuerdo con los planteamientos de la TSD, consideramos fundamental indagar los conocimientos matemáticos de los sujetos en el marco de una tarea determinada, la cual debe tener relación con la noción matemática implicada (Brousseau, 2007). La TSD ofrece un marco teórico que nos permite indagar conocimientos matemáticos específicos, delimitados por una situación determinada. Particularmente el concepto de "variable didáctica", acuñado en la TSD, nos resulta sumamente fructífero para nuestros propósitos.

Las variables didácticas se conciben como aquellas características de un problema que pueden modificarse y tienen un efecto cualitativo importante sobre las evoluciones de los procedimientos (Brousseau, 1981). Son los elementos que "afectan a la jerarquía de estrategias de solución que pone en funcionamiento el alumno (por el costo, la validez, por la complejidad, etc.)" (Brian y Chevalier, 
1995: 68). Con base en lo anterior, diseñamos una tarea para indagar las ideas de los niños acerca del sistema de numeración decimal; establecimos determinadas variables didácticas en esa tarea y anticipamos ciertas respuestas por parte de los alumnos.

La tarea estuvo compuesta por cinco sumas horizontales. Elegimos la presentación de los sumandos de manera horizontal porque, de acuerdo con Broitman, Grimaldi y Ponce (2013), esta presentación favorece reflexiones en torno al valor posicional y a las reglas de la escritura numérica, aspectos donde el uso del cero cobra sentido. Además, de acuerdo con Lerner (1992), plantear a los niños situaciones experimentales donde se les permita reflexionar sobre el valor del cero en el sistema de numeración decimal, genera importantes movilizaciones de conocimiento.

Cada una de las sumas horizontales fue diseñada considerando ciertas variables didácticas, que describimos a continuación.

a) Orden de los sumandos. Los sumandos de la operación se presentaron en dos modalidades: de acuerdo con el orden canónico (unidades de millar, centenas, decenas y unidades simples) o en un orden diferente del canónico. Anticipamos que la diferente presentación de los órdenes tendría efecto en los procedimientos de los alumnos. Podría ser que algunos de los procedimientos atendieran a la cantidad de ceros de cada sumando para determinar a qué orden pertenecen.

b) Ausencia de algún orden en los sumandos. Se omitió algún sumando de cierto orden, por ejemplo, de las centenas. Ello implicó que, en el resultado, esa ausencia de orden se representara con cero. La escritura del cero podría propiciar reflexiones en torno a su función en las escrituras numéricas.

c) Cálculo con agrupamiento decimal o sin agrupamiento decimal. Se incluyeron dos sumandos del mismo orden en el cálculo. Esta característica hizo necesario un proceso de agrupamiento decimal. Es relevante identificar cómo los alumnos justificaron estos procedimientos y qué características de la operación y de los sumandos los conducían a tomar esas decisiones.

d) Cero en el resultado. Centrándonos en el resultado de la suma, teníamos operaciones que daban como resultado una cantidad con cero, ya sea por ausencia de algún orden en la suma o porque, después de hacer todas las agrupaciones decimales no quedaba ninguna unidad de cierto orden. 
Como puede notarse, en cada una de las variables descritas establecimos ciertas hipótesis respecto a las respuestas que podrían dar los alumnos. Recurrimos a las fases de análisis a priori y a posteriori de la metodología de la ingeniería didáctica. De acuerdo con Artigue (1995:45):

...el objetivo del análisis a priori es determinar en qué las selecciones hechas permiten controlar los comportamientos de los estudiantes y su significado. Por lo anterior, este análisis se basa en un conjunto de hipótesis. La validación de estas hipótesis está, en principio, indirectamente en juego en la confrontación que se lleva a cabo en la cuarta fase entre el análisis a priori y el análisis a posteriori.

Por último, elaboramos un análisis posterior, en el cual retomamos las respuestas y producciones de los estudiantes y las analizamos e interpretamos a la luz de nuestros antecedentes y de las predicciones de respuestas consideradas en el análisis previo.

Como mencionamos, lo que aquí se presenta es parte de un estudio (Ávalos, 2016) cuyo propósito fue identificar conocimientos de 10 alumnos de sexto grado respecto al valor posicional. Para ello les planteamos tareas de cálculo aditivo y una de las consignas fue que describieran qué uso daban al cero al resolver dichas tareas.

El trabajo que presentamos aquí reporta, justamente, funciones atribuidas al cero. El objetivo de este artículo es, por una parte, destacar los conocimientos que pone de manifiesto un grupo de escolares de sexto grado sobre el cero al resolver cálculos tipo sumas horizontales; por otra, verificar el impacto de ciertas variables didácticas en los procedimientos de los estudiantes.

\section{MÉTODO}

Dado que el interés de este trabajo se centró en conocer de manera profunda las respuestas de los alumnos, elegimos un diseño de investigación cualitativo. La indagación fue efectuada mediante una entrevista clínica semiestructurada, ${ }^{4}$

4 El objetivo de esta técnica es encontrar el significado de los comportamientos del sujeto y reconstruir el modelo mental que guía las acciones del sujeto ante la tarea planteada por el investigador. Consiste en preguntas básicas comunes para todos los sujetos que se amplían y completan en función de las respuestas de los entrevistados que buscan interpretar lo mejor posible lo que están diciendo (Delval, 2001). 
mediada por un instrumento de investigación que consistió en cinco sumas horizontales. Cada una de las cinco operaciones fue diseñada considerando ciertas variables didácticas.

La muestra fue no probabilística por conveniencia, conformada por 10 estudiantes de una escuela pública de un municipio conurbado de la ciudad de Querétaro, en México. La edad promedio de los alumnos fue de 12 años, y al momento de la entrevista cursaban el último bimestre del sexto grado de primaria.

Entregamos a cada niño una hoja con las operaciones o sumas horizontales y se les pidió que las resolvieran como ellos supieran (la entrevista fue individual). Una vez que el alumno escribía o decía su respuesta para cada operación, la entrevistadora le solicitaba escribir su resultado y justificar sus procedimientos de resolución, así como dar explicaciones sobre el significado del cero. Le recordamos al lector que la investigación original estuvo centrada en los conocimientos de los estudiantes sobre el valor posicional, pero si la investigadora hipotetizaba que el alumno estaba recurriendo a nociones donde se involucraban los ceros, planteaba a los niños más preguntas al respecto, tal como el ejercicio de la entrevista clínica contempla.

\section{ANÁLISIS A PRIORI}

El análisis a priori es una herramienta metodológica que permite, por un lado, describir las características de las situaciones diseñadas y, por otro, anticipar algunos comportamientos de los estudiantes al resolver la situación. En ese sentido, retomamos este tipo de análisis para describir las variables didácticas de nuestra tarea y, con base en esa descripción, anticipar los procedimientos que los alumnos pondrían en juego.

Se hizo un análisis a priori de cada una de las sumas horizontales propuestas. Este análisis permitió, por una parte, anticipar los posibles procedimientos de los niños y, por otra, ayudó a identificar el potencial de la suma para hacer emerger conocimientos de los estudiantes sobre el valor posicional y el cero. El análisis previo de cada suma proporcionó un referente para contrastarlo después con las respuestas que efectivamente dieron los alumnos (análisis posterior). Las siguientes tablas detallan el análisis previo de cada ítem. 
Tabla 1. Análisis previo del ítem 1.

\begin{tabular}{|l|l|l|l|}
\hline Ítem 1 & Objetivo & Posibles procedimientos & $\begin{array}{l}\text { Conocimientos } \\
\text { implicados }\end{array}$ \\
\hline $5000+300+60+4=$ & $\begin{array}{l}\text { Identificar la relación que los } \\
\text { niños establecen entre los } \\
\text { ceros de los sumandos y el } \\
\text { orden al que corresponden } \\
\text { (unidades, decenas, centenas, } \\
\text { etc.). }\end{array}$ & $\begin{array}{l}\text { Los ceros del sumando mayor } \\
\text { podrían ser utilizados por los } \\
\text { alumnos como marcas de } \\
\text { posición de las cifras diferen- } \\
\text { tes de cero de los sumandos } \\
\text { de menor orden. }\end{array}$ & $\begin{array}{l}\text { El ceromo que sirve } \\
\text { cifra indicar un } \\
\text { espacio vacío } \\
\text { en la escritura } \\
\text { polinominal. }\end{array}$ \\
\hline $\begin{array}{l}\text { Variables didácticas } \\
\text { Orden canónico de los sumandos: esta operación se caracteriza por la presentación de sumandos } \\
\text { en orden de mayor a menor, correspondiente a la descomposición decimal canónica en unidades } \\
\text { de millar, centenas, decenas, unidades. }\end{array}$ \\
\hline $\begin{array}{l}\text { Preguntas para profundizar en las respuestas de los niños } \\
\text { ¿Qué resultado obtuviste? } \\
\text { ¿En qué te fijaste para llegar a ese resultado? } \\
\text { ¿De qué te sirven los ceros? }\end{array}$ \\
\hline
\end{tabular}

Tabla 2. Análisis previo del ítem 2.

\begin{tabular}{|c|c|c|c|}
\hline Ítem 2 & bjetivo & Posibles procedimientos & \\
\hline \multirow[t]{2}{*}{$2000+80+2=$} & \multirow[t]{2}{*}{$\begin{array}{l}\text { Indagar qué función } \\
\text { atribuían los sujetos al } \\
\text { cero del resultado de } \\
\text { la operación (2082). }\end{array}$} & \multirow{2}{*}{$\begin{array}{l}\text { Los ceros del sumando mayor } \\
\text { podrían servir de modelo para } \\
\text { escribir los demás sumandos, } \\
\text { pero con las dificultades de } \\
\text { tener que elegir dónde escribir } \\
\text { el cero. }\end{array}$} & $\begin{array}{l}\text { El cero como cifra que } \\
\text { sirve para indicar un } \\
\text { espacio vacío en la } \\
\text { escritura polinominal. }\end{array}$ \\
\hline & & & \\
\hline \multicolumn{4}{|c|}{$\begin{array}{l}\text { Variables didácticas } \\
\text { Orden canónico de los sumandos: este ítem presenta los sumandos del orden mayor al menor. } \\
\text { Tiene sumandos de tres órdenes diferentes: millares, decenas y unidades. } \\
\text { Ausencia de algún orden con cero y cero en el resultado: se consideró la ausencia de centenas, } \\
\text { lo cual se expresa con un cero en el resultado de la suma. Sobre el cero, los alumnos tienen que } \\
\text { elegir dónde escribirlo a partir del resultado de su cálculo. }\end{array}$} \\
\hline \multicolumn{4}{|c|}{$\begin{array}{l}\text { Preguntas para profundizar en las respuestas de los niños } \\
\text { ¿Qué resultado obtuviste? } \\
\text { ¿En qué te fijaste para llegar a ese resultado? } \\
\text { En tu resultado yo veo un cero, ¿̇ué significa? } \\
\text { ¿Por qué escribes el cero en ese lugar (dentro de su escritura del resultado) y no en otro sitio? }\end{array}$} \\
\hline
\end{tabular}


Tabla 3. Análisis previo del ítem 3.

\begin{tabular}{|c|c|c|c|}
\hline Ítem 3 & Objetivo & $\begin{array}{l}\text { Posibles } \\
\text { procedimientos }\end{array}$ & $\begin{array}{l}\text { Conocimientos } \\
\text { implicados }\end{array}$ \\
\hline $300+4000+80+3+40=$ & $\begin{array}{l}\text { Este cálculo tuvo como obje- } \\
\text { tivo indagar la relación que } \\
\text { los sujetos podrían estable- } \\
\text { cer entre el número de ceros } \\
\text { de cada sumando y el orden } \\
\text { al que pertenecen. }\end{array}$ & $\begin{array}{l}\text { Que los alumnos iden- } \\
\text { tifiquen los ceros de los } \\
\text { sumandos del mismo } \\
\text { orden y decidan operar- } \\
\text { los en primer lugar. }\end{array}$ & $\begin{array}{l}\text { El cero como indi- } \\
\text { cador del orden al } \\
\text { que pertenecen } \\
\text { los sumandos. }\end{array}$ \\
\hline \multicolumn{4}{|c|}{$\begin{array}{l}\text { Variables didácticas } \\
\text { Orden diferente del canónico: lo que caracteriza a esta operación es que los sumandos no } \\
\text { presentan un orden creciente ni decreciente. } \\
\text { Agrupamiento decimal: además, hay dos sumandos del orden de las decenas que se distinguen } \\
\text { por tener la misma cantidad de ceros. Ello puede usarse como una señal de que ambos suman- } \\
\text { dos se asocien para llevar a cabo el reagrupamiento decimal y obtener el resultado de la suma. }\end{array}$} \\
\hline \multicolumn{4}{|c|}{$\begin{array}{l}\text { Preguntas para profundizar en las respuestas de los niños } \\
\text { ¿Qué resultado obtuviste? } \\
\text { ¿En qué te fijaste para llegar a ese resultado? } \\
\text { En caso de que asocien las decenas para operarlas: ¿Por qué decides sumar el } 80 \text { y el } 40 \text { aparte? }\end{array}$} \\
\hline
\end{tabular}

Tabla 4. Análisis previo del ítem 4.

\begin{tabular}{|l|l|l|l|}
\hline Ítem 4 & Objetivo & $\begin{array}{l}\text { Posibles } \\
\text { procedimientos }\end{array}$ & $\begin{array}{l}\text { Conocimientos } \\
\text { implicados }\end{array}$ \\
\hline $600+5000+7+400+40=$ & $\begin{array}{l}\text { En esta tarea se } \\
\text { buscó conocer la } \\
\text { función atribuida } \\
\text { por los alumnos al } \\
\text { cero en el resultado } \\
\text { (6047), como resul- } \\
\text { tado de una agru- } \\
\text { pación decimal. }\end{array}$ & $\begin{array}{l}\text { Que los alumnos identi- } \\
\text { fiquen los ceros de los } \\
\text { sumandos del mismo } \\
\text { orden y decidan operar- } \\
\text { los en primer lugar. }\end{array}$ & $\begin{array}{l}\text { El cero como indica- } \\
\text { dor del orden al que } \\
\text { pertenecen los su- } \\
\text { mandos. }\end{array}$ \\
& $\begin{array}{l}\text { El cero como cifra } \\
\text { que sirve para indi- } \\
\text { car un espacio vacío } \\
\text { en la escritura poli- } \\
\text { nominal. }\end{array}$ \\
\hline $\begin{array}{l}\text { Variables didácticas } \\
\text { Orden diferente del canónico: los sumandos no se presentan en orden canónico. En este } \\
\text { cálculo existirá un cero en el resultado, como en el ítem 2. }\end{array}$ \\
$\begin{array}{l}\text { Agrupamiento decimal y cero en el resultado: la diferencia con el ítem } 2 \text { es que en este cálculo } \\
\text { el cero no representa directamente la ausencia de unidades en el orden de las centenas, sino } \\
\text { que resulta de un proceso de transformación de centenas en unidades de millar. }\end{array}$ \\
\hline
\end{tabular}




\section{Preguntas para profundizar en las respuestas de los niños}

¿Qué resultado obtuviste?

¿En qué te fijaste para llegar a ese resultado?

Se previeron intervenciones como las siguientes: "Veo que en tu resultado hay un cero, ¿qué quiere decir ese cero?". Si los alumnos argumentaban que no había centenas, les hacíamos ver que entre los sumandos había algunas centenas como 600 y 400, planteando preguntas como la siguiente: "Yo veo que hay centenas y tú me dices que el cero indica que no hay centenas, ¿cómo es eso?".

Tabla 5. Análisis previo del ítem 5.

\begin{tabular}{|c|c|c|c|}
\hline Ítem 5 & Objetivo & $\begin{array}{l}\text { Posibles } \\
\text { procedimientos }\end{array}$ & $\begin{array}{l}\text { Conocimientos } \\
\text { implicados }\end{array}$ \\
\hline \multirow[t]{3}{*}{$9000+1700+800+5=$} & \multirow{3}{*}{$\begin{array}{l}\text { Con esta operación se } \\
\text { indagaron las reglas de la } \\
\text { escritura numérica que } \\
\text { incluyen al cero, relacio- } \\
\text { nadas con los procesos de } \\
\text { transformación de agrupa- } \\
\text { mientos o agrupación } \\
\text { decimal. }\end{array}$} & \multirow{3}{*}{$\begin{array}{l}\text { Que los alumnos } \\
\text { identifiquen los ceros } \\
\text { de los sumandos del } \\
\text { mismo orden y deci- } \\
\text { dan operarlos en } \\
\text { primer lugar, con el } \\
\text { reto que representa } \\
\text { desagrupar un suman- } \\
\text { do (1700) para rea- } \\
\text { gruparlo y obtener el } \\
\text { resultado. }\end{array}$} & $\begin{array}{l}\text { El cero como indi- } \\
\text { cador del orden al } \\
\text { que pertenecen los } \\
\text { sumandos. }\end{array}$ \\
\hline & & & $\begin{array}{l}\text { El cero como cifra } \\
\text { que sirve para } \\
\text { indicar un espacio } \\
\text { vacío en la escritura } \\
\text { polinominal. }\end{array}$ \\
\hline & & & $\begin{array}{l}\text { El cero como } \\
\text { diferenciador de } \\
\text { cantidades. }\end{array}$ \\
\hline \multicolumn{4}{|c|}{$\begin{array}{l}\text { Variables didácticas } \\
\text { Orden canónico, cero en el resultado y agrupamiento decimal: los sumandos se presentan en } \\
\text { orden canónico. En este cálculo existirá un cero en el resultado, como en el ítem } 2 \text {. Sin } \\
\text { embargo, la diferencia con el ítem } 2 \text { es que en este cálculo el cero no representa directamente } \\
\text { la ausencia de unidades en el orden de las centenas, sino que resulta de dos procesos de } \\
\text { transformación de centenas en unidades de millar y de unidades de millar a decenas de millar. } \\
\text { La diferencia entre esta operación y la anterior viene dada por el rango numérico y por un } \\
\text { sumando que se presentó agrupado (1700). Supusimos que estas características podrían tornar } \\
\text { más compleja esta operación respecto a las anteriores. }\end{array}$} \\
\hline \multicolumn{4}{|c|}{$\begin{array}{l}\text { Preguntas para profundizar en las respuestas de los niños } \\
\text { ¿Qué resultado obtuviste? } \\
\text { ¿En qué te fijaste para llegar a ese resultado? } \\
\text { En tu resultado veo un cero, ¿qué significa ese cero? }\end{array}$} \\
\hline
\end{tabular}




\section{DATOS Y ANÁLISIS POSTERIOR}

Obtuvimos 10 entrevistas completas, con numerosas respuestas para cada uno de los 5 ítems de la tarea. A través de un estudio interpretativo efectuado en el programa de análisis cualitativo ATLAS.ti se obtuvieron 35 respuestas sobre las funciones del cero, las cuales constituyeron nuestra unidad de análisis.

Las respuestas de los alumnos fueron organizadas en categorías; para ello retomamos funciones y usos del cero ya identificados en la literatura y citados en este documento: el cero como diferenciador de cantidades y el cero como representación de ausencia.

Dado que en nuestro estudio encontramos respuestas que no se alineaban completamente con las categorías anteriormente mencionadas, fue necesario proponer una categoría más: el cero como indicador de cómo sumar. A continuación, describimos las categorías en las que se agruparon las respuestas.

A) El cero como indicador de cómo sumar: los estudiantes se fijan en el número de ceros de cada sumando y deciden un procedimiento de resolución del cálculo.

B) El cero como diferenciador de cantidades: Ios entrevistados indican que el cero se escribe para que un número no sea confundido con otro.

C) El cero como representación de ausencia: los alumnos señalan que el cero se escribe cuando no hay algún sumando de cierto orden, o para guardar el espacio de algún orden.

\section{RESULTADOS}

Como mencionamos, el objetivo de este artículo es destacar los conocimientos que tiene un grupo de escolares de sexto grado sobre el cero al resolver cálculos tipo sumas horizontales, y verificar el impacto de ciertas variables didácticas en los procedimientos de los alumnos. A continuación presentamos las categorías en las que se han agrupado las respuestas y que dan cuenta de los conocimientos que subyacen a las mismas. Más adelante describimos las variables didácticas de las tareas y la forma en la que impactaron las respuestas. 


\section{DESCRIPCIÓN DE CADA UNA DE LAS CATEGORÍAS}

La Tabla 6 muestra la frecuencia de aparición de respuestas relacionadas con el cero, organizadas en las categorías anteriormente citadas.

Tabla 6. Frecuencia de respuestas por categoría en cada ítem.

\section{Categoría}

\begin{tabular}{lccc}
\hline \multicolumn{1}{c}{ Ítem } & $\begin{array}{c}\text { Indicador } \\
\text { de cómo sumar }\end{array}$ & $\begin{array}{c}\text { Diferenciador } \\
\text { de cantidades }\end{array}$ & $\begin{array}{c}\text { Representación } \\
\text { de ausencia }\end{array}$ \\
\hline $5000+300+60+4=$ & 3 & & 1 \\
$2000+80+2=$ & 4 & 3 & 7 \\
$300+4000+80+3+40=$ & 1 & & \\
$600+5000+7+400+40=$ & 3 & 1 & 5 \\
$9000+1700+800+5=$ & 2 & 2 & 3 \\
\hline Total & 13 & 6 & 16
\end{tabular}

Es importante señalar que las categorías sobre las funciones del cero identificadas en los alumnos no son excluyentes, pues en ocasiones un mismo alumno hizo alusión a más de una función en una misma respuesta, como podrá apreciarse en algunos de los ejemplos que presentamos.

\section{a) El cero como indicador de cómo sumar}

En 13 ocasiones los estudiantes atendieron al número de ceros de cada sumando para elegir un procedimiento de cálculo. Así, el número de ceros fue referencia directa del orden de cada sumando, tras lo cual ellos decidieron cómo llevar a cabo el cálculo. Hubo dos modalidades en esta categoría.

La primera de ellas (aplicada por cuatro niños en los dos primeros ítems) implica que atendieron los ceros del sumando mayor para "acomodar" sobre ellos las cifras diferentes a cero de los sumandos menores. Constatamos que, tal como habíamos anticipado, esos alumnos utilizaron los ceros para sobre escribir las unidades de cada orden. Por ejemplo, en la operación 5000+300+60+4= 
acomodaron el 3 sobre el cero que guarda la posición de las centenas, el 6 sobre el cero de las decenas y el 4 en el lugar de las unidades del 5000 (Figura 2).

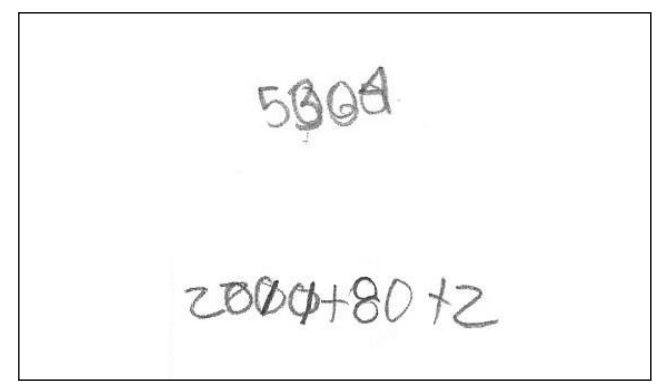

Figura 2. Uso de los ceros como guías para acomodar el resultado del cálculo.

La segunda modalidad se presentó cuando los estudiantes señalaron que los ceros de los sumandos les indicaron "cómo sumar". Fue aplicada a partir del ítem 3 (que implica transformación de unidades). En estos casos la estrategia de 4 alumnos fue identificar el orden decreciente y la agrupación decimal de cada sumando con base en los ceros. Teniendo en cuenta esta información, los entrevistados notaron la necesidad de llevar a cabo la reagrupación decimal. Por ejemplo, en la operación $600+5000+7+400+40=$ estos niños se percataron de que 400 y 600 tienen el mismo número de ceros y, por tanto, debe llevarse a cabo una reagrupación de centenas a millares. Así lo explica Dulce: ${ }^{5}$

Entrevistador: Me cuentas que sumaste seiscientos y cuatrocientos, ¿en qué te fijaste para sumar esos dos?

Dulce: Yo siempre me fijo en si tiene tres ceros y son cinco mil, aquí son dos ceros y son seiscientos y aquí pues también tiene dos ceros.

Complementando este razonamiento otra alumna, Karen, indicó que para resolver la operación "tiene que sumar todos con ceros...", "pues que sume todo con ceros... porque los ceros también valen ¿no?".

En esta segunda modalidad los estudiantes usaron la cantidad de ceros de un numeral para tomar decisiones sobre cómo reordenar los sumandos para

\footnotetext{
5 Los nombres han sido cambiados para proteger la identidad de los participantes.
} 
operarlos; también decidieron si es conveniente o no adicionar como primer paso del cálculo aquellos sumandos del mismo orden (decenas o centenas primero). Resulta interesante que, para los entrevistados que dieron este tipo de respuestas, los ceros son signos que deben ser considerados cuando se está resolviendo una operación. Parece que este acomodo de ceros tiene similitud con el space filler referido por Blake y Verhille (1985). En estos casos, aunque los ceros no se operen para obtener el resultado, el lugar que ocupan acompaña los pasos de un procedimiento de solución.

\section{b) El cero como diferenciador de cantidades}

En seis de las respuestas analizadas, los alumnos indicaron que la escritura del cero en un determinado lugar de un numeral, lo diferencia de otro. Por ejemplo, en la operación 2000+80+2=2082 señalaron que el cero en 2082 se escribe para que el número sea "dos mil ochenta y dos" en lugar de "doscientos ochenta y dos" o "dos mil ochocientos veinte".

La alumna Karen refirió que el cero diferencia las cantidades de 647 y 6047. Además, explicitó la necesidad de escribir ceros para que el numeral sea del orden de los millares.

Entrevistador: Oye, ¿̇y qué significa que ese cero esté ahí? [El cero de 6047].

Karen: Que equivale a seis mil, si le quito el cero nada más me va a dar seiscientos cuarenta y siete. Equivale a mil.

En estos registros, la escritura del cero aparece como relevante para ubicar al número en otro orden. A estas ideas puede subyacer la noción del cero como elemento que resguarda una potencia de base. Si se elimina el cero, se elimina la potencia, y con ello el valor de todo el número (Lerner, 1992). La entrevistada Yarah lo expone de la siguiente manera: "Pues el cero debe estar aquí porque si quito el cero serían once mil cincuenta y cinco, entonces si no valiera el cero, pues sería otro resultado y el cero en este momento pues sí vale porque el cero está respetando el número; por decir, si aquí le pongo un seis ya sería el sesenta y cinco. El cero está marcando, o sea para que pueda dar otro resultado".

Recordemos que, históricamente, diferenciar cantidades fue el problema que originó la invención del cero. 


\section{c) El cero como representación de ausencia de unidades de algún orden}

En 16 respuestas los alumnos argumentaron que el cero se escribe para indicar que no hay algún sumando de cierto orden. Por ejemplo, en el resultado de 2082 señalaron que el cero se escribe porque "no hay centenas" en la suma $2000+80+2=$.

La afirmación anterior no es estrictamente convencional, pues en el sumando 2000 sí hay 20 centenas, pero asumimos que los niños centraron su atención en los sumandos de la operación y no en los numerosos agrupamientos posibles que pueden hacerse con dos mil unidades.

Relacionado con lo anterior, cuando los estudiantes resolvieron cálculos con reagrupación decimal pudieron ofrecer respuestas donde no sólo indican que el cero representa unidades de cierto orden, sino que también resulta que "ya no quedan decenas" o "centenas" formadas en el proceso del cálculo. Fue en el marco de estas tareas cuando los alumnos manifestaron concepciones del cero como representación de ausencia, haciendo alusión explícita a que el cero representa la ausencia de algún orden.

Al respecto, nos interesó identificar la explicitación de conceptos y reglas del sistema decimal por parte de los alumnos. Los ejemplos que presentamos a continuación dan cuenta de ello.

Los estudiantes explicaron que el cero marca o respeta una posición que puede ser ocupada por unidades de algún orden. Si bien tal explicación indica una ausencia, como en el caso previo, este tipo de razonamiento se diferencia del anterior porque está orientado al establecimiento de que el cero puede cambiarse por unidades de cualquier orden, una vez que se formen en el continuo proceso de transformación decimal.

Por ejemplo, de los diez entrevistados, cinco niños hicieron referencia a la posición al contestar a la pregunta “ipor qué escribes un cero en tu resultado (2082)?”. Estas respuestas estuvieron relacionadas con la representación de ausencia de centenas agrupadas en el ítem 2000+80+2=. Es decir, los alumnos indicaron que el cero se escribía en ese lugar porque era el que deberían ocupar las centenas:

Samantha: El cero va porque todavía no llega a tener centena, entonces pues se deja el espacio de la centena y ya se pone a partir de la decena.

Interpretamos que Samantha señala el cero como un "espacio" que sería llenado por centenas en caso de que las hubiera, lo cual coincide, además, con el uso 
del cero para representar ausencia; parece coincidir también con el uso del cero como "variable", usado para representar lo desconocido en una ecuación hasta que sea ocupado por un número determinado (Blake y Verhille, 1985), o como "vacío receptor" (Kaplan, 2004). Con ello, la entrevistada muestra una concepción sobre la agrupación decimal como una transformación continua y no estática, cuando indica que "todavía no llega a tener centena".

En posteriores intervenciones pudimos constatar que Samantha argumenta coordinando dos ideas: el cero como representación de ausencia y el cero como marca de posición. Lo interesante de esta respuesta es la claridad con la que indica el proceso de transformación entre órdenes y cómo este proceso se vincula a la escritura numérica del resultado, que incluye ceros en este caso.

(Refiriéndose al cero en 11505)

Entrevistador: Ok, ¿cómo decides escribir ese cero en ese lugar?

Samantha: Porque aquí pues ya estaban estos hasta las centenas [Refiriéndose a que ha calculado $9000+1700+800=11500$; pero le falta sumar 5 unidades] y después se les suma el cinco, entonces con el cinco ya no alcanza a las decenas, entonces se deja el espacio de las decenas con cero y ya se pasan las unidades.

Queremos enfatizar que esta última categoría contribuye a la comprensión de la evolución del concepto cero en el contexto del sistema decimal. Como mencionamos, el cero tiene la dualidad de representar a la vez ausencia de unidades y presencia de una posición. Por tanto, el que algunos de nuestros entrevistados pudieran explicitar que un cero se escribe para "respetar" el lugar de algún orden que no se agrupó en el proceso de cálculo, resultó sumamente valioso para los propósitos de nuestro análisis.

\section{PAPEL DE LAS VARIABLES DIDÁCTICAS}

En el análisis previo anticipamos que las variables de orden de los sumandos, ausencia de algún orden, agrupamiento decimal y cero en el resultado tendrían impacto en los procedimientos. Enseguida se describen las implicaciones que tales variables tuvieron en las respuestas de los estudiantes. 
En $37.14 \%$ de las respuestas, los niños usaron los ceros como apoyo para elegir un procedimiento de cálculo. Esta variable tuvo dos modalidades: presentar los sumandos en el orden canónico (descendente) y en un orden diferente del canónico. Cada una de esas modalidades propició procedimientos diferentes.

Orden canónico. La presentación de los sumandos de la forma millares, centenas, decenas, unidades generó en los alumnos un procedimiento de cálculo donde los ceros del sumando mayor fueron modelo para escribir las cifras diferentes de cero de los sumandos de orden menor.

Orden diferente del canónico. Esta presentación de los sumandos provocó que los estudiantes se apoyaran en los ceros para saber la agrupación decimal a la que pertenecía cada sumando, y así poder decidir un procedimiento de cálculo. Cabe señalar que esta modalidad no se distingue, en cuanto a sus efectos, de la variable didáctica denominada "cálculo con o sin agrupamiento decimal". Entonces, más que afirmar que esta no tuvo efectos, pareciera que, metodológicamente, no se distingue de la presentación de sumandos en orden diferente del canónico. En todo caso, queda pendiente de análisis.

En $17.14 \%$ de las respuestas, la variable de ausencia de algún orden con cero en el resultado sí tuvo efecto: propició que, al escribir los resultados, los alumnos enfrentaran alguna dificultad al decidir dónde escribir el cero; también dio pie a reflexiones en torno al significado del cero en las escrituras numéricas.

Respecto a estos ítems donde el cero figuró en el resultado de la suma, obtuvimos $45.71 \%$ de respuestas donde los niños explicaron el significado del cero. Este hecho enfatiza que tareas con las características descritas pueden ser fructíferas para propiciar la reflexión sobre la función del cero en el sistema decimal. Podemos afirmar, entonces, que algunas de las variables didácticas elegidas tuvieron los efectos esperados.

\section{DISCUSIÓN}

Es notable la diversidad de funciones que los alumnos atribuyen al cero. En cuanto al primero de esos usos -el cero para calcular- cobra relevancia el procedimiento por el cual los niños se apoyaron en los ceros del sumando mayor para componer el resultado (ponen sobre los ceros del sumando mayor las cifras diferentes de cero de los sumandos menores). Esta forma de resolución ya ha sido reportada por Lerner (2005) y Delprato (2002). 
En el trabajo de Lerner los sujetos que participaron en su estudio (estudiantes de segundo grado) no pudieron vincular el hecho de "tachar los ceros" con las reglas de la numeración escrita ni con el valor posicional. En cambio, los niños de nuestro estudio (alumnos de sexto grado) sí relacionaron explícitamente los ceros del sumando mayor con marcas de posición, además de que usaron los ceros para determinar los agrupamientos decimales a los que correspondía cada sumando. Apoyados en estos saberes han establecido reglas de la escritura numérica del sistema decimal. Al parecer, la diferencia en cuanto a los años de escolaridad de ambas muestras incide en la diferencia de conocimientos que se pusieron de manifiesto.

Hay otro aspecto relevante en este procedimiento: los alumnos de sexto grado pudieron expresar tres condiciones que, a su juicio, tendría que cumplir una operación para poder colocar las cifras diferentes del cero de los sumandos menores, sobre los ceros del sumado mayor. Las dos primeras condiciones cumplen el carácter de necesarias para poder usar este procedimiento: primero, hay que acomodar los sumandos de manera decreciente (por ejemplo, millares, centenas, decenas, unidades); segundo, hay que asegurarse de tener sólo un sumando de cada orden para acomodar sin problema las cifras diferentes de cero sobre los ceros del sumando mayor. La tercera condición no es indispensable, sin embargo, también fue expresada por los estudiantes: no deben faltar sumandos de algún agrupamiento (como el caso las centenas en 2000+80+2=). No es indispensable, puesto que si no hay algún sumando -las centenas, en el caso del ejemplo- eso se indica con el cero.

Por su parte, Delprato (2002) encontró un procedimiento equivalente al anterior en una investigación llevada a cabo con adultos no alfabetizados. En ese trabajo los sujetos usaron los números con ceros (en nuestro caso, los sumandos mayores) como modelos para escribir sobre sus ceros las cifras diferentes de cero restantes.

Tenemos entonces que para los alumnos de segundo grado (Lerner, 2005), para los estudiantes de sexto grado de nuestro estudio y para los adultos no alfabetizados del estudio de Delprato (2002), la información que los sujetos reciben de los ceros se transforma en una vía de solución. Sin embargo, la diferencia relevante es que los niños de sexto grado explicaron por qué funciona ese procedimiento, poniéndolo en relación con propiedades del sistema de numeración decimal.

Respecto a los ítems que implican transformación de orden, los ceros de los sumandos indicaron a los alumnos la agrupación decimal a la cual 
corresponden. Una vez identificados dos sumandos del mismo orden, optaron por sumarlos para obtener un nuevo orden, mediante una transformación. Ese procedimiento puede parecer obvio, pero nos resulta interesante saber cuáles son los criterios de los niños para proceder de tal forma. Ellos argumentan haberse fijado en el número de dígitos y en los ceros de los sumandos para optar por un procedimiento de resolución. Tal argumento nos lleva a apoyar el planteamiento de algunos autores sobre la poca conveniencia de enseñar el algoritmo de suma en columnas, particularmente en etapas tempranas de la educación primaria (Kamii y Joseph, 1990; Lerner, 1992). El algoritmo en columnas está diseñado de tal manera que la transformación decimal se expresa en términos de unidades y no de agrupamientos íntegros, por lo que su enseñanza en fases tempranas de la educación básica podría conducir a obstáculos en la comprensión del valor posicional.

En nuestro trabajo encontramos algunos entrevistados que usaron el cero para diferenciar cantidades. Este uso parece coincidir en cuanto a su definición con el cero placeholder de Blake y Vermhill (1985) o cero cifra de D'Amore y Fandiño (2014). Pese a que es la función histórica más primitiva del cero, consideramos que apoyarse en los ceros para interpretar correctamente cantidades es un recurso plenamente justificado. Este conocimiento permite a los alumnos responder uno de los muchos retos que representa escribir números con ceros. Los estudios de Wolman y Ponce (2013) y Centurión y Saiz (2014) refuerzan nuestra posición. En ellos, los autores reportan que estudiantes que ya dominan una parte importante de la serie numérica, siguen creando reglas originales para expresar e interpretar números grandes (del orden de centenas de millar y millones). Para los niños de sus investigaciones, los ceros son una importante señal de cómo leer un número y sirven para saber su valor. En cuanto a los ceros para diferenciar e interpretar cantidades, sus estudios y el nuestro coinciden.

Sostenemos que plantear tareas y preguntas que involucran trabajar con el cero realmente problematizó a los entrevistados. Ello confirma los argumentos de Lerner (1992) y Martí (2005) respecto a que plantear problemas con cero conduce, por un lado, a movilizar conocimientos y, por otro, a vencer obstáculos cognitivos que implican representar una ausencia mediante una presencia.

Consideramos que los alumnos de nuestra investigación dan cuenta de haber superado dicha dicotomía, pues reconocen que el cero ocupa un espacio que puede ser ocupado por elementos de determinada potencia de base (unidades, decenas, centenas, etc.). Para que lo anterior se manifestara fue relevante la estructura de cada operación: por ejemplo, cuando los niños resolvieron una 
operación como $2000+80+2=2082$ indicaron que el cero del resultado quiere decir que "no hay decenas"; pero cuando resuelven operaciones con transformación, como $600+4000+7+400+40=6047$ y se les plantea la misma pregunta, los estudiantes indican que el cero es el resultado de haber transformado las centenas a millares.

Este matiz en las respuestas de los alumnos da cuenta del efecto que tiene variar la estructura de las operaciones. Cuando la operación hacía evidente la transformación, se movilizaron conceptos del cero no sólo como representación de ausencia, sino como representación de ausencia de una posición. Este conocimiento representa, según Martí (2005), la consolidación en el proceso de aprendizaje del sistema de numeración decimal.

\section{CONCLUSIONES}

El objetivo de esta investigación fue identificar cuál es la función que los estudiantes de sexto grado de primaria asignan al signo cero y cómo lo usan para resolver tareas de cálculo. Por otro lado, también nos planteamos indagar el efecto de las variables didácticas en las respuestas de los niños.

Los alumnos de este estudio pudieron desplegar numerosos conocimientos sobre el cero. Usaron los ceros para decidir procedimientos de cálculo, para diferenciar números; saben que el cero representa ausencia de unidades de algún orden y a la vez saben, y esto es lo más relevante para nosotros, que ese vacío puede ser llenado en cualquier momento.

Al respecto, encontramos respuestas de algunos niños que explicaron el cero en términos de "marca de una posición". Para ellos, el cero se pone en el lugar en el cual potencialmente podrían ir las unidades, decenas, centenas, etc., que no hay por el momento. Consideramos que esta manera de concebir el cero es un rasgo que caracteriza el pensamiento matemático, puesto que implica desprenderse de la tarea concreta y empezar a interpretar los números como parte de un sistema donde cualquier número puede ser representado.

Las variables didácticas elegidas al diseñar cada ítem de nuestro instrumento son prometedoras en cuanto a que propiciaron la presencia de diferentes estrategias de resolución y, a su vez, permitieron recurrir a explicaciones sobre los distintos sentidos del cero.

En la revisión curricular encontramos que, si bien el estudio del cero se propone de manera indirecta al abordar las diferencias entre sistemas numéricos, 
en general las actividades propuestas sobre el cero no son suficientemente sistemáticas. Consideramos que otra vía de entrada a su estudio puede ser a través de los cálculos del tipo que presentamos en esta exploración. Además, podemos afirmar entonces que las variables didácticas elegidas permitieron desplegar numerosos conocimientos y generar algún tipo de dificultad que puede aprovecharse en las aulas para generar conflictos cognitivos y, con ello, progresos en las nociones matemáticas sobre el cero en la estructura del sistema de numeración decimal.

Por último, resaltamos la importancia de los errores y los conflictos que manifestaron los niños en las tareas que implicaron la escritura del cero en el resultado (2082, 6047 o 11505). Coincidimos en este punto con lo planteado por Lerner (1992): introducir situaciones que consideren reflexionar sobre el cero puede problematizar los esquemas de conocimiento de los alumnos y, a la vez, hacerlos avanzar. En esa dirección, creemos que si bien nuestro estudio no explora situaciones de enseñanza, los resultados del mismo son una invitación para que en el aula se propongan múltiples tareas que impliquen escribir ceros y operar con ellos. La invitación también incluye plantear reflexiones en torno a los diversos significados del cero y a la historia misma de ese signo, pues todo ello contribuye a que los estudiantes den sentido al "vacío" que el cero representa.

Proponemos, para estudios posteriores, una investigación sistemática acerca del desarrollo de las nociones sobre el cero en diversos grupos de edad. Así también, mejorar el diseño de las variables didácticas y profundizar el estudio de su impacto en los procedimientos de los alumnos. Un estudio más específico sobre la compatibilidad y equivalencia de las funciones del cero propuestas por diferentes autores.

\section{Agradecimientos}

Para la elaboración de la investigación que aquí reportamos, se contó con una beca del Consejo Nacional de Ciencia y Tecnología (Conacyt). Número de beca 330192. 


\section{REFERENCIAS}

Anthony, G. y Walshaw, M. (2004). Zero: a "None Number"? Teaching Children Mathematics, 11(1), 38-42.

Artigue, M. (1995). Ingeniería didáctica. En Gómez, P. (ed.). Ingeniería didáctica en educación matemática (pp. 33-69). Bogotá: Grupo Editorial Iberoamérica.

Ávalos, O. (2016) Conocimientos sobre valor posicional en alumnos de sexto grado de primaria (Tesis de maestría no publicada). Universidad Autónoma de Querétaro. México.

Blake, R. y Verhille, C. (1985). The Story of 0. For the Learning of Mathematics, 5(3), 35-47. Brian, J. y Chevalier, M. (1995). Les enjeux didactiques dans l'enseignements des mathématiques. París: Hatier.

Broitman, C, Grimaldi, V. y Ponce, H. (2011). El valor posicional. Reflexiones y propuestas para su enseñanza. Bueno Aires: Santillana.

Brousseau, G. (1981). Problèmes de didactique des décimaux (Fregona, D. y Soto, R. trans.). Recherches en Didactique des Mathématiques, 2(1), 37-128.

Brousseau, G. (2007). Iniciación al estudio de la teoría de las situaciones didácticas. Buenos Aires: Libros del Zorzal.

Brown, M. (1981). Number Operations. En Hart, K. (ed.), Children's Understanding of Mathematics (pp. 23-47). London: John Murray.

Centurión, L. y Saiz, I. (noviembre de 2014) Conocimientos, herramientas de control y conflictos en la producción de escrituras de números grandes en el nivel secundario. En Saiz, I. La enseñanza de la numeración, la suma y la resta en el primer ciclo de la Educación Básica. Conferencia llevada a cabo en la Subsecretaría de Educación Básica de la Secretaría de Educación Pública. Ciudad de México.

Crooks, T. y Flockton, L. (2001). Mathematics Assessment Results 2001. National Education Monitoring. Dunedin, New Zealand: Educational Assessment Research Unit, University of Otago.

D’Amore, B. y Fandiño, M. (2014). El número cero. Aspectos históricos, epistemológicos, filosóficos, conceptuales y didácticos del número más misterioso. México: Neisa.

Delprato, M. F. (2002). Los adultos no alfabetizados y sus procesos de acceso a la simbolización matemática (Tesis de maestría no publicada). CINVESTAV-IPN. México.

Delval, J. (2001). Descubrir el pensamiento de los niños: introducción a la práctica del método clínico. España: Siglo XXI.

García-Milà, M., Teberosky y Martí, E. (2000). Anotar para resolver un problema de localización y memoria. Infancia y Aprendizaje, 90, 2000, 51-70.

Hughes, M. (1986). Children and Number: Difficulties in Learning Mathematics. Nueva York: Basin Blackwell. 
Ifrah, G. (1987). Las cifras. Historia de una gran invención. Madrid: Alianza Editorial.

Kamii, C. y Joseph, L. (1990). La enseñanza del valor posicional y de la adición en dos columnas. Comunicación, Lenguaje y Educación, 2(6), 27-35.

Kaplan, R. (2004). Una historia natural del cero. La nada que existe. México: Océano. Leeb-Lundberg, K. (1977). Zero. Mathematics Teaching, 78, 24-25.

Lerner, D. (1992). El valor posicional. En Lerner, D. (ed.). La matemática en la escuela aquí y ahora (pp. 155-207). Bueno Aires: Aique.

Lerner, D. (2005). ¿Tener éxito o comprender? Una tensión constante entre la enseñanza y el aprendizaje del sistema de numeración. En Alvarado, M, y Brizuela, B. (comps.). Haciendo números. Las notaciones numéricas vistas desde la psicología, la didáctica y la historia (pp. 147-197). México: Paidós.

Lerner, D. y Sadovsky, P. (1994). El sistema de numeración: un problema didáctico. En Saiz, I. y Parra, C. (comps.). Didáctica de matemáticas. Aportes y reflexiones. Buenos Aires: Paidós.

Lovell, K. (1971). The growth of understanding in mathematics: Kindergarten through grade three. New York: Holt, Rinehart \& Winston.

Martí, E. (2005). Las primeras funciones de las notaciones numéricas. Una mirada evolutiva. En Alvarado, M. y Brizuela, B. (comps.). Haciendo números. Las notaciones numéricas vistas desde la psicología, la didáctica y la historia (pp. 51-80). México: Paidós.

Scheuer, N., Sinclair, A., De Rivas, S. M. y Christinat, C. T. (2000). Cuando ciento setenta y uno se escribe 10071: niños de 5 a 8 años produciendo numerales. Infancia y Aprendizaje, 23(90), 31-50.

SEP (2011). Plan de estudios 2011. Educación básica. México: SEP.

Wellman, H. y Miller, K. (1986). Thinking about nothing: Development of concepts of zero. Britsh Journal of Developmental Psychology, 4(1), 31-42.

Wolman, S. y Ponce, H. (2013). Relaciones entre la escritura de números y su designación oral: el uso de puntos en niños que ya dominan un rango importante de la serie. En Broitman, C. (comp.). Matemáticas en la escuela primaria (1). Números naturales y decimales con niños y adultos (pp. 203-229). Buenos Aires: Paidós.

OLIVIA ÁvALOS ESPARZA

Dirección: Av. Santuario de Guadalupe \#984 interior 54, Paseos del Bosque, CP. 76910 Corregidora, Querétaro.

Teléfono: $\quad 4427040072$ 